March 1993

PUPT-1391

\title{
Logarithmic Operators in Conformal Field Theory
}

\author{
V. Gurarie \\ Department of Physics \\ Princeton University \\ Princeton, NJ 08544
}

\begin{abstract}
Conformal field theories with correlation functions which have logarithmic singularities are considered. It is shown that those singularities imply the existence of additional operators in the theory which together with ordinary primary operators form the basis of the Jordan cell for the operator $L_{0}$. An example of the field theory possessing such correlation functions is given.
\end{abstract}




\section{Introduction}

One of the basic assumptions of Conformal Field Theory is the existence of the space of fields with the operator product expansion. The coefficients of this expansion are supposed to be powers of the distance and it can be used to construct all the correlation functions. For example, the expansion of the four point correlation function of some operators in powers of the anharmonic ratio exactly corresponds to the operator product expansion of those operators. In many interesting cases the Laurent series expansion exists. But it turns out there are some cases when this expansion does not exist, which means the operator product expansion as it is understood in Conformal Field Theory must be modified.

Let us review some of the results obtained in [1] which we will need. One of them was the existence of the so called primary operators $A_{k}(z)$. These operators transform under conformal transformations in a certain way, namely when $z \rightarrow f(z)$,

$$
A_{k}(z) \rightarrow\left(\frac{d f}{d z}\right)^{h_{k}} A_{k}(f)
$$

where $h_{k}$ is called the (right) dimension of the operator $A_{k}$.

The requirement of the correlation functions of the theory to be conformaly invariant provides certain restrictions on the correlation functions. In particular, the two point correlation function of two primary operators must behave as a power of distance,

$$
<A_{k}(z) A_{k}(w)>=\frac{\text { const }}{(z-w)^{2 h_{k}}}
$$

The three point correlation functions are also fixed up to a constant. As for the four point correlation functions of the primary operators, they are fixed up to an arbitrary function of the so called anharmonic ratio,

$$
<A_{k}\left(z_{1}\right) A_{k}\left(z_{2}\right) A_{k}\left(z_{3}\right) A_{k}\left(z_{4}\right)>=\frac{1}{\left(z_{1}-z_{3}\right)^{2 h_{k}}\left(z_{2}-z_{4}\right)^{2 h_{k}}} F(x)
$$

where $F(x)$ is some function and $x$ is the anharmonic ratio

$$
x=\frac{\left(z_{1}-z_{2}\right)\left(z_{3}-z_{4}\right)}{\left(z_{1}-z_{3}\right)\left(z_{2}-z_{4}\right)}
$$

It is well-known that the expansion of $F(x)$ in powers of $x$ corresponds to the operator product expansion of Conformal Field Theory. Conformal invariance requires the operator product expansion to have the form

$$
B_{k}(z) B_{j}(w)=\sum_{n} \frac{C_{n}}{(z-w)^{h_{k}+h_{j}-h_{n}}} B_{n}(w)
$$

where $B$ are some (not only primary) operators. On the other hand if we substitute this expansion to (3), we will get exactly the Laurent series expansion of the function $F(x)$. 
The function $F(x)$ is not easy to determine. In most cases, only its Laurent series expansion coefficients can be calculated one after another, as was shown in [1]. But in the cases of the so called degenerate operators correlation functions are known to satisfy certain differential equations. We can determine $F(x)$ by solving these equations. It turns out these equations are the differential equations for the generalized hypergeometric functions [2]. So, for the degenerate operator four point correlation functions $F(x)$ are the generalized hypergeometric functions. In most cases, these functions can be expanded in Laurent series around $x=0$. But sometimes for the special values of the operator dimensions the functions $F(x)$ can have logarithmic divergencies near $x=0$. In fact, the most general behavior for $F(x)$ near $x=0$ is given by

$$
F(x)=\sum_{n=0}^{\infty} a_{n} x^{n}+\log (x) \sum_{n=0}^{\infty} b_{n} x^{n}+\log ^{2}(x) \sum_{n=0}^{\infty} c_{n} x^{n}+\ldots
$$

Such a behavior is in explicit contradiction with the operator product expansion (5).

We will have to introduce new operators in the operator product expansion, operators which will have "logarithmic" behavior. Ordinary primary operators are known to be the eigen vectors of the $L_{0}$ operators, and their eigen values are the dimensions of these operators. It will be shown that those "new" operators, which I will call pseudo-operators, are the basis of the Jordan cell for $L_{0}$.

As we will see, the logarithm-like singularities in the correlation functions are quite a rare occasion in conformal field theories. That's probably why they have not been studied before even though the logarithms in the correlation functions of WZW models have already appeared in [3]. They do not appear in unitary minimal models, and only nonminimal or possibly nonunitary minimal models have a chance of possessing them. However, such nonminimal models have recently emerged in the studies of percolations [4] and polymers [5]. Also, the recent work on conformal turbulence [6] has revived the interest in nonunitary minimal models and provided us with new motivations to understand them better. We hope this paper contributes to that understanding.

\section{Logarithmic pseudo operators}

To begin with, we consider the case of the $c=-2$ model. It provides us with the example of the most simple correlation function with logarithmic behavior. Really, let us

take the operator $(1,2)$ with dimension $-\frac{1}{8}$. We will call it $\mu$ from now on. If we try to compute the four point correlation function of the operator $\mu$

$$
<\mu\left(z_{1}\right) \mu\left(z_{2}\right) \mu\left(z_{3}\right) \mu\left(z_{4}\right)>=\left(z_{1}-z_{3}\right)^{\frac{1}{4}}\left(z_{2}-z_{4}\right)^{\frac{1}{4}}[x(1-x)]^{\frac{1}{4}} F(x)
$$

we discover that the function $F(x)$ satisfies the following differential equation as a consequence of the degeneracy of $\mu$ on the second level.

$$
x(1-x) \frac{d^{2} F(x)}{d x^{2}}+(1-2 x) \frac{d F(x)}{d x}-\frac{1}{4} F(x)=0
$$


We want to find the asymptotic behavior of $F(x)$ as $x \rightarrow 0$. Substituting $F(x) \sim x^{\alpha}$ and taking the leading term we get $\alpha^{2}=0$. Both two solutions of this equation are $\alpha=0$. The standard interpretation of this fact by [1] would be that both operators arising in the Operator Product Expansion of $\mu(z) \mu(w)$ have dimension 0, so it seems they are the same unit operators. But this in fact is incorrect. If these two $\alpha$ were different, we would really obtain the asymptotic behavior of two linear independent solution of (8) corresponding to the contributions of two different primary operators and their descendants. But since they are the same, simple analysis of (8) shows that while the first solution of (8) really behaves as $x^{0}$ the other solution behaves as $\log (x)$.

One might think that one can simply discard the second solution of (8) and do not worry about logarithms. But this is not true. It is not difficult to check that the first solution of (8) which is regular at $x=0$ will have a logarithmic singularity at $x=1$. Therefore, it is impossible to get rid of the logarithms in the OPE of the operators $\mu$.

Let us see how this works. We look for the first solution of (8) in terms of the series

$$
\sum_{n=0}^{\infty} a_{n} x^{n}
$$

After substituting it to (8) one can find the coefficients $a_{n}$ one after another recursively. It gives us the first solution of (8) regular at $x=0$. In fact, it is going to be the hypergeometric function $F\left(\frac{1}{2}, \frac{1}{2} ; 1 ; x\right)$, but this is not important for us at the moment. Now to look for the second solution of (8) we consider the following candidate

$$
\sum_{n=0}^{\infty} b_{n} x^{n} \log (x)+c_{n} x^{n}
$$

The first thing we see after substituting it to (8) is $b_{n}=a_{n}$. Then $c_{n}$ can also be obtained one after another.

Fortunately, the equation (8) is simple enough for its exact solution to exist in a closed form. It can be expressed in terms of the elliptic integral

$$
G(x) \equiv \int_{\varphi=0}^{\frac{\pi}{2}} \frac{d \varphi}{\sqrt{1-x \sin ^{2}(\varphi)}}
$$

and is given by

$$
F(x)=C_{1} G(x)+C_{2} G(1-x)
$$

$C_{1}$ and $C_{2}$ are the arbitrary constants. $G(x)$ is regular at $x=0$. The behavior of $G(1-x)$ can be understood if we use the following formula

$$
G(1-x)=G(x) \log (x)+H(x)
$$

where $H(x)$ is some regular at $x=0$ function. (10) of course follows immediately from the above discussion of the solution properties. A very important point which must be 
emphasized now is that the logarithmic solution of (8) has the regular solution of (8) multiplied by the logarithm plus some other regular function.

We are going to construct the operator product expansion of $\mu(z) \mu(0)$ compatible with the solutions of (8). The first regular solution of (8) implies the ordinary OPE

$$
\mu(z) \mu(0)\left|>=z^{\frac{1}{4}}\right| I, z>
$$

where $|I, z\rangle$ is the contribution of the identity operator and its descendants (we will work with the states instead of the operators for a moment; $\mid>$ is the vacuum). The second solution of (8) corresponds to a new OPE

$$
\mu(z) \mu(0) \mid>=z^{\frac{1}{4}}\left(\log (z)|I, z>+| I_{1}, z>\right)
$$

where $\mid I_{1}, z>$ denotes the contribution of the new pseudo-operator with unusual properties.

The dilatation transformation performed on (12) hints we must have

$$
L_{0} I_{1}=I
$$

This can be done more rigorously by the method developed in [1]. Namely let us expand $\mid I, z>$ and $\mid I_{1}, z>$ in series

$$
\left|I, z>=\sum_{n=0}^{\infty} z^{n}\right| I, n>
$$

and

$$
\left|I_{1}, z>=\sum_{n=0}^{\infty} z^{n}\right| I_{1}, n>
$$

substitute these series to (12) and apply $L_{k}$ to both sides of the equality obtained. Then the standard procedure usually used to determine coefficients in the conformal block and including the commuting of $L_{k}$ with $\mu(z)$ and using

$$
\left[L_{k}, A(z)\right]=\left(z^{k+1} \frac{\partial}{\partial z}+(k+1) h_{A} z^{k}\right) A(z)
$$

for any primary operator $A(z)$ will give

$$
\begin{gathered}
L_{0}|I, n>=n| I, n> \\
L_{0}\left|I_{1}, n>=\right| I, n>+n \mid I_{1}, n> \\
L_{k}\left|I, n+k>=\left(n+(k-1) h_{\mu}\right)\right| I, n> \\
L_{k}\left|I_{1}, n+k>=\right| I, n>+\left(n+(k-1) h_{\mu}\right) \mid I_{1}, n>
\end{gathered}
$$

$h_{\mu}=-\frac{1}{8}$ is the dimension of $\mu, k>0$ in (14).

These equations accumulate all the properties of the operator $I_{1}$. First we see that

$$
L_{0}\left|I_{1}, 0>=\right| I, 0>
$$


as we expected. So, the operators $I_{1}$ and $I$ are the basis of the Jordan cell for $L_{0}$. Secondly, we can explicitly write

$$
\begin{gathered}
\left|I_{1}, 1>=\beta_{1} L_{-1}\right| I_{1}, 0> \\
\mid I_{1}, 2>=\beta_{2} L_{-2}\left(a|I, 0>+| I_{1}, 0>\right)+\beta_{11}\left(L_{-1}\right)^{2}\left(b|I, 0>+| I_{1}, 0>\right)
\end{gathered}
$$

and so on, then the equations (14) help us to determine all $\beta$ 's, $a, b$, etc. ${ }^{\dagger}$

It is also useful to see how conformal bootstrap can be solved in this case. Following [2] we must construct a single-valued function of $x$ and $\bar{x}$ out of the solutions of (8) which will give us the "full" correlation function, with its holomorphic and antiholomorphic dependence. Using (10) we can check that the answer is

$$
\left(z_{1}-z_{3}\right)^{\frac{1}{4}}\left(z_{2}-z_{4}\right)^{\frac{1}{4}}[x(1-x)]^{\frac{1}{4}} \overline{\left(z_{1}-z_{3}\right)^{\frac{1}{4}}\left(z_{2}-z_{4}\right)^{\frac{1}{4}}[x(1-x)]^{\frac{1}{4}}}[G(x) G(1-\bar{x})+G(\bar{x}) G(1-x)]
$$

This answer is different from the ordinary case of "normal" operators, the usual answer would be $\sum_{k} G_{k}(z) G_{k}(\bar{z})$ while here we have something like $G_{1}(z) G_{2}(\bar{z})+G_{2}(z) G_{1}(\bar{z}), G_{k}$ are the linear independent solutions for the correlation function differential equation. We interpret this fact by noting that the "full" operators in the theory must have a normal holomorphic and a pseudo antiholomorphic dimension or vice versa.

Now we can generalize the above scheme. Consider the model of the conformal field theory where there are two operators OPE of which, found according to the rules of [1], contains at least two operators with the same dimensions. As we know now, that means these rules are no longer valid and in fact these two operators are the basis of the Jordan cell for $L_{0}$. So, let

$$
A(z) B(0)=z^{h_{C}-h_{A}-h_{B}}\left\{C_{1}+\ldots+\log (z)(C+\ldots)\right\}
$$

After repeating the same arguments we arrive at the following formulae

$$
\begin{gathered}
L_{k}\left|C, n+k>=\left(h_{C}+n-h_{B}+k h_{A}\right)\right| C, n> \\
L_{k}\left|C_{1}, n+k>=\right| C, n>+\left(h_{C}+n-h_{B}+k h_{A}\right) \mid C_{1}, n> \\
L_{0}\left|C, n>=\left(h_{C}+n\right)\right| C, n> \\
L_{0}\left|C_{1}, n>=\right| C, n>+\left(h_{C}+n\right) \mid C_{1}, n>
\end{gathered}
$$

which are, not surprisingly, the straightforward generalizations of (13) and (14). Using (19) and (19) we can express $\mid C, n>$ and $\mid C_{1}, n>$ in terms of the descendants of $C$ and $C_{1}$ the same way as we have done already for the case of $I$ and $I_{1}$. The answer for the first level is

$$
\begin{gathered}
\left|C, 1>=\frac{h_{C}+h_{A}-h_{B}}{2 h_{C}} L_{-1}\right| C, 0> \\
\left|C_{1}, 1>=\frac{h_{C}+h_{A}-h_{B}}{2 h_{C}} L_{-1}\right| C_{1}, 0>+\frac{h_{B}-h_{A}}{2 h_{C}^{2}} L_{-1} \mid C, 0>
\end{gathered}
$$

It may be interesting to try to find correlation function containing the operator $C_{1}$. We can proceed here in many different ways. For example, we can take the correlation function

$$
<A\left(z_{1}\right) B\left(z_{2}\right) A\left(z_{3}\right) B\left(z_{4}\right)>
$$

$\dagger$ We must include descendants of $\mid I, 0>$ into $\mid I_{1}, k>$ for consistency. Let us note that it doesn't contradict (13). We do not include $L_{-1} \mid I, 0>$ to $\mid I_{1}, 1>$ as $L_{-1} \mid I, 0>=0$. 
and expand the product $A\left(z_{3}\right) B\left(z_{4}\right)$ according to (17). On the other hand, we know this function is going to behave like

$$
\frac{1}{\left(z_{1}-z_{3}\right)^{h_{A}}} \frac{1}{\left(z_{2}-z_{4}\right)^{h_{B}}} \frac{1}{[x(1-x)]^{h_{A}+h_{B}-h_{C}}}\left[\log (x)\left(a_{0}+a_{1} x+\ldots\right)+b_{0}+b_{1} x+\ldots\right]
$$

So, if we take this expression, substitute the value for $x$ in terms of $z_{1}, z_{2}, \ldots$, and extract from it a coefficient multiplying $\left(z_{3}-z_{4}\right)^{h_{C}-h_{A}-h_{B}}$, we will get exactly

$$
<A\left(z_{1}\right) B\left(z_{2}\right) C_{1}\left(z_{3}\right)>=<A\left(z_{1}\right) B\left(z_{2}\right) C\left(z_{3}\right)>\left[\log \frac{z_{1}-z_{2}}{\left(z_{1}-z_{3}\right)\left(z_{2}-z_{3}\right)}+\lambda\right]
$$

This function turns out to be invariant with respect to the translation, dilatations, rotations and special conformal transformations. ${ }^{\dagger}$ The appearance of the arbitrary parameter $\lambda$ is due to the fact that we can always replace $C_{1} \rightarrow C_{1}+\lambda C$.

We can continue the expansion of (21) to get two point correlation functions. which turn out to be

$$
\begin{gathered}
<C_{1}(z) C_{1}(w)>=-\frac{2}{(z-w)^{2 h_{C}}}\left[\log (z-w)+\lambda^{\prime}\right] \\
<C(z) C_{1}(w)>=\frac{1}{(z-w)^{2 h_{C}}}
\end{gathered}
$$

It is not possible to determine $\lambda$ 's without knowing the structure constants of the operator algebra.

Another way to find the correlation functions is to study the operator product expansion of $C(z) C_{1}(w), A(z) C_{1}(w)$ etc. by the method used to derive (13) and (14). Let us again consider for simplicity the case of $c=-2$. We want, for example, to find the operator product expansion of $\mu(z) I_{1}(w)$. To do that, first we denote

$$
\mu(z) I(0)|>=| \mu, z>_{1}
$$

The next step will be to assume that

$$
\mu(z) I_{1}(0)|>=\log (z)| \mu, z>_{2}+\mid \mu, z>_{3}
$$

We denote here the blocks by $\left|\mu, z>_{1},\right| \mu, z>_{2}$, and $\mid \mu, z>_{3}$ to emphasize that the coefficients of the expansions in terms of the descendants of $\mu$ in these blocks may be different. And the last step is to apply $L_{k}, k \geq 0$ to both sides of this equation. Taking into account the unusual property of $I_{1}$, namely that $L_{0} I_{1}=I$, we get

$$
\begin{gathered}
\left(z \frac{\partial}{\partial z}+h_{\mu}\right)\left(\log (z)\left|\mu, z>_{2}+\right| \mu, z>_{3}\right)+\mid \mu, z>_{1}= \\
\left(\log (z) L_{0}\left|\mu, z>_{2}+L_{0}\right| \mu, z>_{3}\right) \\
\left(z^{k+1} \frac{\partial}{\partial z}+(k+1) h_{\mu} z^{k}\right)\left(\log (z)\left|\mu, z>_{2}+\right| \mu, z>_{3}\right)= \\
\log (z) L_{k}\left|\mu, z>_{2}+L_{k}\right| \mu, z>_{3}
\end{gathered}
$$

$\dagger$ When checking this statement, one has to keep in mind that the transformation law for $C_{1}$ is given by $\delta C_{1}(z)=\epsilon^{\prime}(z)\left[h_{C} C_{1}(z)+C(z)\right]+\epsilon(z) C_{1}^{\prime}(z)$ 
$k>0$

It is clear from (25) and (26) is $\left|\mu, z>_{2}=-\right| \mu, z>_{1}$. Then the structure of $\mid \mu, z>_{3}$ can also be determined. So, we proved that

$$
\mu(z) I_{1}(0)|>=-\log (z) \mu(z) I(0)|>+\mid \mu, z>_{3}
$$

$\mid \mu, z>_{3}$ is a conformal block different from the one arising in the expansion of $\mu(z) I(0) \mid>$.

Similar technique can be applied to find any OPE in the theory with pseudo-operators. These OPE will be compatible with the correlation functions (21) and (22).

Our construction can be naturally generalized to the case of three or more operators forming the basis of the Jordan cell for $L_{0}$. Then we have $m$ operators $C_{n}$ such that

$$
\begin{gathered}
L_{0} C_{n}=h_{C} C_{n}+C_{n-1}, n>0 \\
L_{0} C_{0}=h_{C} C_{0}
\end{gathered}
$$

and they enter the OPE in the following form

$$
\begin{gathered}
A(z) B(0)=z^{h_{C}-h_{B}-h_{A}}\left\{C_{0}+\ldots\right\} \\
A(z) B(0)=z^{h_{C}-h_{B}-h_{A}} \log (z)\left\{C_{0}+\ldots\right\}+z^{h_{C}-h_{B}-h_{A}}\left\{C_{1}+\ldots\right\} \\
\ldots \ldots \ldots \ldots \ldots \ldots \ldots \ldots \ldots \ldots \ldots \ldots \ldots \ldots \ldots \ldots \ldots \ldots \ldots \ldots \\
A(z) B(0)=z^{h_{C}-h_{B}-h_{A}} \sum_{n} \log ^{n}(x)\left\{C_{m-n}+\ldots\right\}
\end{gathered}
$$

From the above discussion it is clear we must include logarithmic operators in the theory if it possesses at least two operators the product of which when expanded according to the fusion rules of [1] contains the contribution of at least two operators with the same dimension. It can be proved such a case never occurs in the minimal models. But it always occurs in any non-minimal model. So, non-minimal models are in fact richer than it was thought they were.

\section{Other representations of the Virasoro algebra.}

There is another case when logarithmic correlation functions occur in the conformal field theory. Namely, it is the case when there are operators in the theory the OPE of which contains operators dimensions of which differ by the integer number. Really, if this is the case, then the equation for the function $F(x)$ defined analogously to $F(x)$ in $(7)$ possesses two asymptotic solutions, $x^{0}$ and $x^{n}, n$ is some positive integer number. Then there are two possibilities. Either the function

$$
F(x)=\sum_{m=0}^{n-1} a_{m} x^{m}
$$

with the appropriate choice of $a_{m}$ is an exact solution of the corresponding differential equation or the two linear independent solutions of this equation are

$$
F(x)=\sum_{m=n}^{\infty} b_{m-n} x^{m}
$$


and

$$
F(x)=\sum_{m=0}^{\infty} c_{m} x^{m}+\log (x) \sum_{m=n}^{\infty} b_{m-n} x^{m}
$$

Here we again have to introduce the logarithmic operators. But their behavior will be more complicated. The solution (31) corresponds to the contribution of the ordinary primary operator while the solution (32) corresponds to the contribution of the same operator multiplied by $\log (x)$ plus the contribution of another pseudooperator with even more unusual properties than the ones considered in section 2 .

Really, this operator must have dimension smaller than the dimension of its ordinary partner, but still the operator $L_{0}$ must again be nondiagonal in the same fashion as it was before. If we suppose that simply $L_{0}\left|C_{1}, m>=\right| C, m-n>+\left(h_{C_{1}}+m\right) \mid C_{1}, m>$ if $m \geq n$ and $L_{0}\left|C_{1}, m>=\left(h_{C_{1}}+m\right)\right| C_{1}, m>$ otherwise, we immediately obtain a contradiction. By applying $L_{-1}$ to

$$
L_{0}\left|C_{1}, n-1>=\left(n-1+h_{C_{1}}\right) L_{-1}\right| C_{1}, n-1>
$$

we get

$$
L_{0} L_{-1}\left|C_{1}, n-1>=\left(n+h_{C}\right) L_{-1}\right| C_{1}, n-1>
$$

which is difficult to satisfy in view of

$$
L_{0}\left|C_{1}, n>=\right| C, 0>+\left(h_{C_{1}}+n\right) \mid C_{1}, n>
$$

We have to use a more complicated construction. Let us make the operator $C_{1}$ have the dimension equal to that of $C$ with the same Jordan-like philosophy, $L_{0} \mid C_{1}, m>=$ $\left|C, n>+\left(m+h_{C}\right)\right| C, m>$ but also let us make the operator $C_{1}$ to be "even less" primary, that is $L_{n}\left|C_{1}>=\right| C^{1}>$ where $\mid C^{1}>$ is a primary operator of the dimension $h_{C}-n$. Then the OPE corresponding to (31) and (32) are

$$
\begin{gathered}
A(z) B(0)=z^{h_{C}-h_{A}-h_{B}}(C(0)+\ldots) \\
A(z) B(0)=z^{h_{C}-h_{A}-h_{B}}\left\{\log (z)(C(0)+\ldots)+\left(C_{1}+\ldots\right)+z^{-n}\left(C^{1}+\ldots\right)\right\}
\end{gathered}
$$

Then we can work out in the same way all the coefficients in the operator product expansion of different operators.

The simplest example (and unfortunately the only one known to me) when all discussed above is the case can be found in the same $c=-2$ theory. If we compute the correlation function of the operators $(1,2)$ and $(2,2)$ of this theory we will obtain

$$
<A_{(1,2)}\left(z_{1}\right) A_{(2,2)}\left(z_{2}\right) A_{(1,2)}\left(z_{3}\right) A_{(2,2)}\left(z_{4}\right)>=\left(z_{1}-z_{3}\right)^{\frac{1}{4}}\left(z_{2}-z_{4}\right)^{-\frac{3}{4}}[x(1-x)]^{\frac{3}{4}} F(x)
$$

where the function $F(x)$ turns out to be the first derivative of (9). So, its expansion around the logarithmic point is given by the derivative of (10),

$$
F(x)=G^{\prime}(x) \log (x)+\frac{G(x)}{x}+H^{\prime}(x)
$$


which is of the same form as (32). We see that the construction of OPE of $A_{(1,2)}(z) A_{(2,2)}(w)$ includes one normal operator $W$ with the dimension 1 , one "pseudo" operator $W_{1}$ with the dimension 1 and the property $L_{0} W_{1}=W+W_{1}$ and one primary operator $W^{1}$ with the dimension 0 and the property $L_{-1} W_{1}=W^{1}$. The behavior of $W^{1}$ will almost always coincide with that of the unit operator, it has the same dimension after all and all $L_{k} W^{1}=$ 0 for $k>0$.

We can proceed to find the OPE of these operators. It will be the same procedure as the one described in section 2. For example, the expansion of $A_{(1,2)}(z) W_{1}(0)$ will be given by

$$
A_{(1,2)}(z) W_{1}(0)\left|>=-z^{-\frac{5}{4}}\left(\log (z)\left|A_{(2,2)}, z>_{1}+\right| A_{(2,2)}, z>_{2}\right)+z^{-1}\right| A_{(1,2)}, z>
$$

where the first two terms on the right hand side are constructed analogously to (27) and the third term satisfies the equations

$$
\begin{gathered}
z^{-1} L_{1}\left|A_{(1,2)}, z>=\left(z^{2} \frac{\partial}{\partial z}+2 h_{A} z\right) z^{-1}\right| A_{(1,2)}, z>+A_{(1,2)}(z) W^{1}(0) \mid> \\
z^{-1} L_{2}\left|A_{(1,2)}, z>=\left(z^{3} \frac{\partial}{\partial z}+3 h_{A} z^{2}\right) z^{-1}\right| A_{(1,2)}, z>
\end{gathered}
$$

The interesting question is whether the case of such logarithmic operators occurs in the minimal models. I do not know yet the answer to this question. All minimal models I studied explicitly so far have been the examples of (30). Nevertheless, the possibility of nonunitary minimal model with correlation functions (31) and (32) cannot be ruled out at this point.

\section{Conclusions}

A physical example of the theory with logarithmic operators can be provided by the free ghost model with the action

$$
S \sim \int \partial \theta \bar{\partial} \bar{\theta} d^{2} z
$$

where $\theta$ and $\bar{\theta}$ are the anticommuting variables. This theory is analogous to the $\eta$, $\xi$ system studied in [5] in connection with polymers.

It is shown in the appendix that the central charge of this theory is equal to $c=-2$ and its physics can be described by the non-minimal model extensively discussed in this paper. In particular, all the operators $(n, 1)$ with the dimensions $\frac{n^{2}-n}{2}$ correspond to

$$
\partial \theta \partial^{2} \theta \ldots \partial^{(n-1)} \theta
$$

or

$$
\partial \bar{\theta} \partial^{2} \bar{\theta} \ldots \partial^{(n-1)} \bar{\theta}
$$

Then the primary operators $(1,2)$ and $(2,2)$ will be twist operators of this theory and their correlation functions will have logarithmic singularities. This implies there are also 
logarithmic pseudooperators in this theory as was shown in the numerous examples in this paper.

It was already mentioned in the Introduction that according to Cardy [4] the correlation function desribing percolations should be taken from the nonminimal $c=0$ theory. Even though this function by itself does not possess logarithmic singularities, other functions of this theory do. The OPE of the operators underlying this theory will also include logarithmic pseudooperators.

So, we have shown some of conformal field theories necessarily possess some other operators in addition to just primary. Unfortunately, the task of classifying all such theories has not been accomplished here. The main difficulty is to find a way to distinguish differential equation with a polynomial solution (30) from that with a logarithmic solution (32). One of the ways to overcome that difficulty is to use explicit formulas for the structure constants of conformal field theory given in [2]. It turns out those formulas give infinity in the case of logarithmic conformal field theories, technically due to gamma functions of negative integers. It would be interesting to carry out such an analysis of those structure constants. It will not be an easy task either, since the structure constants are given by long and ugly expressions.

\section{Appendix}

We are going to study the free ghost model described by the action (36) to show that it is described by the $c=-2$ minimal model.

With the appropriate normalization of the action, the correlation function is given by

$$
<\partial \theta(z) \partial \bar{\theta}(w)>=-\frac{1}{(z-w)^{2}}
$$

Then the stress-energy tensor is given by

$$
T(z)=: \partial \theta(z) \partial \bar{\theta}(z):
$$

and the central charge is equal to $c=-2$.

Let us consider the twist fields of this theory. Let

$$
\partial \theta(z)=\sum_{n} \theta_{n} z^{-n-1}, \partial \bar{\theta}(z)=\sum_{n} \bar{\theta}_{n} z^{-n-1}
$$

Then

$$
\left\{\theta_{n} \bar{\theta}_{m}\right\}=-n \delta_{n+m, 0}
$$

\{\} means anticommutator. Then we assume $n, m \in \mathbf{Z}+\frac{1}{2}$. To study the properties of the twist fields obtained we have to impose the OPE of $\partial \theta$ with those fields. If we assume $\partial \theta(z)|\sigma>\sim \sqrt{z},| \sigma>$ is the state assosiated with the corresponding twist field, then

$$
\theta_{n} \mid \sigma>=0, n \geq-\frac{1}{2}
$$


It gives rise to

$$
<\sigma|\partial \theta(z) \partial \bar{\theta}(w)| \sigma>=-\sum_{n=\frac{3}{2}, \frac{5}{2}, \ldots} n w^{n-1} z^{-n-1}=-\left(\frac{1}{(z-w)^{2}}+\frac{1}{2 z(z-w)}\right) \sqrt{\frac{w}{z}}(a .4)
$$

Now if $w=z+\epsilon$ then

$$
<\sigma|\partial \theta(z) \partial \bar{\theta}(z+\epsilon)| \sigma>\approx-\frac{1}{\epsilon^{2}}+\frac{3}{8 z^{2}}
$$

which means

$$
L_{0}\left|\sigma>=\frac{3}{8}\right| \sigma>
$$

Quite analogously, $\partial \theta(z) \mid \mu>\sim \sqrt{\frac{1}{z}}$ gives

$$
<\mu|\partial \theta(z) \partial \bar{\theta}(w)| \mu>=-\frac{z+w}{2(z-w)^{2} \sqrt{z w}}
$$

which gives

$$
L_{0}\left|\mu>=-\frac{1}{8}\right| \mu>
$$

The dimensions of the fields $\mu, \sigma$ and $\partial \theta$ coincide with the dimensions of the fields $A_{(1,2)}, A_{(2,2)}$ and $A_{(2,1)}$ of the conformal model $c=-2$. It is alluring to conclude they are the same fields. To prove it, all we have to do is to compare the correlation functions of those fields.

It is straightforward to check that the correlation functions of the operators $A_{(2,1)}$ are the same as those of $\partial \theta$ or $\partial \bar{\theta}$. Actually, to make the correspondence even more clear, the following construction is useful. We can identify

$$
A_{(2,1)} \sim \sigma_{x} \partial \theta+\sigma_{y} \partial \bar{\theta}
$$

where $\sigma_{x}$ and $\sigma_{y}$ are Pauli matrices. The OPE of those operators will be given by

$$
\left(\sigma_{x} \partial \theta(z)+\sigma_{y} \partial \bar{\theta}(z)\right)\left(\sigma_{x} \partial \theta(0)+\sigma_{y} \partial \bar{\theta}(0)\right)=\sigma_{z}(I+\ldots)+\left(\partial^{2} \theta(0) \partial \theta(0)+\partial^{2} \bar{\theta}(0) \partial \bar{\theta}(0)+\ldots\right)
$$

The first brackets symbolize the contribution of the unit operator, while the second ones show the contribution of the operator $A_{(3,1)}$ with the dimension 3 . This example shows how the series of the operators (37) discussed in the Conclusions section has appeared.

It is no more difficult to calculate the correlation functions with $A_{(1,2)}$ and $A_{(2,2)}$. We get

$$
\lim _{\substack{z_{4} \rightarrow 0 \\ z_{2} \rightarrow \infty}} z_{2}^{-\frac{1}{4}}<A_{(2,1)}\left(z_{1}\right) A_{(1,2)}\left(z_{2}\right) A_{(2,1)}\left(z_{3}\right) A_{(1,2)}\left(z_{4}\right)>=\frac{1}{\left(z_{1}-z_{3}\right)^{2}} \frac{z_{1}+z_{3}}{\sqrt{z_{1} z_{3}}}
$$


which coincides with (a.5) up to a constant.

The analogous calculations prove the same for the fields $A_{(2,2)}$ and $\sigma$.

Now we are allowed to calculate the correlation functions $\langle\mu \mu \mu \mu\rangle$ using the rules of conformal field theory. As we already know those functions will have logarithmic singularities which means there are logarithmic operators in the OPE of $\mu$ 's and $\sigma$ 's. Let us again stress that even though we do not know how to construct them explicitly we can always draw the following logical line: free ghosts $\rightarrow$ twisted correlation functions $\rightarrow$ twist fields $\rightarrow$ their correlation functions $\rightarrow$ logarithmic pseudooperators.

\section{Acknowledgements.}

The author is grateful to A.M.Polyakov for encouragement during this work and to A. Matytsin and D. Polyakov for stimulating discussions.

\section{References}

[1] A. Belavin, A. Polyakov and A. Zamolodchikov, Nucl. Phys. B241, 33 (1984).

[2] Vl.S. Dotsenko and V.A. Fateev Nucl. Phys. B240, 312 (1984).

[3] L. Rozansky, H. Saleur Nucl. Phys. B376, 461 (1992)

[4] J. Cardy, Preprint UCSBTH-91-56 (1991)

[5] H. Saleur, Preprint YCTP-P38-91 (1991)

[6] A.Polyakov, Preprint PUPT-1369 (1992) 